\title{
Nothing like the Sun
}

\author{
On 21 August, for those lucky enough to be in the right place at the right time, nothing will happen for a \\ few blissful minutes, and it will mean everything.
}

A total solar eclipse occurs every 18 months or so somewhere on Earth, but this month's event will span the United States from coast to coast - the first in 99 years to do so. Twelve million people live within the $100-110 \mathrm{~km}$-wide path of totality, where it will be safe to view the wisps of the corona with the naked eye for a few minutes (pictured), and millions more are within a few hours' drive. In fact, more than half the population of the US lives within $650 \mathrm{~km}$ of the path of totality; all will have at least a partial eclipse, weather permitting. And if the sky is clear, the experience will be unforgettable - not just a visible corona in a dark sky but a concomitant drop in temperature accompanied by creature silence (Frank Close describes the feeling in Eclipse: Journeys to the Dark Side of the Moon; article no. 0045). It is an outreach opportunity on a silver platter.

And indeed NASA has been busy. Their dedicated website, https://eclipse2017.nasa. gov, includes information on safe viewing as well as the science behind eclipses and doing science during eclipses. They even list several citizen science programs that the public can participate in "at every level from the most basic observations to publishable research opportunities in partnership with NASA and university scientists". And they don't stop with the solar eclipse. There are also studies of solar physics, the Moon and eclipses in other stellar systems in which they invite amateurs to participate.

Not to be outdone, the American Astronomical Society has put together its eclipse website, https://eclipse.aas.org, with the tagline "Sun... Moon... You!" Besides plenty of resources that you would expect, it even has information for people who need to book accommodation. They have also awarded 31 grants up to US $\$ 5,000$ to fund eclipse outreach activities, particularly those focusing on women/girls, ethnic minorities, and people with physical and/or mental disabilities.

There are countless other online resources (including from the International Astronomical Union; http://www.eclipses.info) for supporting scientists, teachers and the public at large, and over 2 million pairs of free eclipseviewing glasses will be distributed by public libraries (courtesy of the Gordon

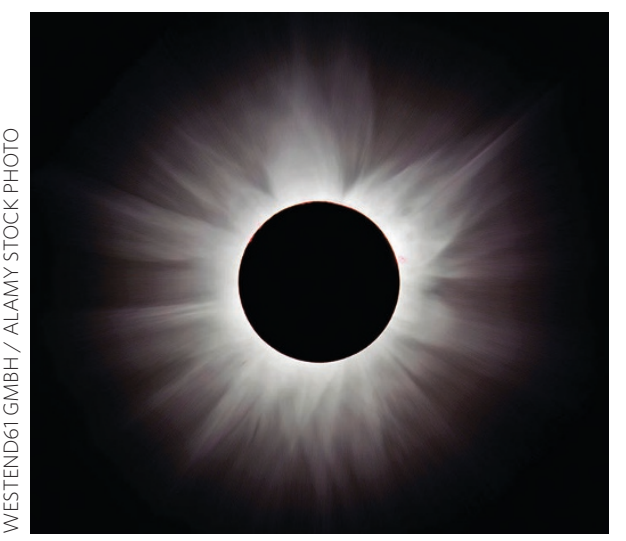

along with scientist Hugh Mortimer and educator Rachel Evans.

Other successful forms of outreach include music, photography, film and books. For example, Garik Israelian started the Starmus Festival in Tenerife, Spain, and the latest one took place in Trondheim, Norway, featuring a clutch of Nobel laureates, astronauts and musicians. And for years, the Royal Observatory, Greenwich, has hosted the Insight Astronomy Photographer of the Year competition, which gets more difficult to judge every year as more entries come in. Last year's winning image was of a series of Baily's beads shot during a total solar eclipse by Yu Jun (http://go.nature.com/2va4HMH).

Additionally, there is a renewed interest of and Betty Moore Foundation). And for those who cannot be 'in the zone', the eclipse will be live-streamed by multiple sources - including from weather balloons at 30,000 metres on NASA TV as well as local and national TV stations.

But besides the pure spectacle of enjoying the beauty of the Sun's corona, scientists have been planning detailed observations to characterize the corona and its dynamics throughout the 11-year solar cycle. For it is only during a solar eclipse that such studies are possible. In this issue, Jay Pasachoff reviews the advances in heliophysics made during eclipses in the past ten years (article no. 0190). He emphasizes the importance of ground-based observations: "no current space coronagraph can observe the inner or middle corona (nor can views from ground-based coronagraphs match the quality of eclipse imaging)". Thus, although eclipses over the South Pacific have their distinct advantages, in terms of setting up equipment, the path of the 2017 eclipse could hardly be closer to ideal for solar physicists.

From previous solar eclipses around the world, we know that the public interest in the 2017 eclipse will be huge. The concerted effort of astronomers and institutions across North America will help inspire a new generation of children to take an interest in the natural world. You don't need to be a scientist to appreciate the wonders of astronomy. Take choreographer Alexander Whitley, for instance, whose dance company performs 8 Minutes, the time it takes light from the Sun to reach our planet (article no. 0196). He discusses his interest in the Sun and astronomy in a Q\&A (article no. 0160) film and TV producers looking to tap into the public's curiosity for science, presenting it in a more rigorous way than in the past. In the film The Martian, for instance, NASA and the Jet Propulsion Laboratory (JPL) are on centre stage, and a Martian Day organized at JPL was attended by tens of thousands of people. Despite some inaccuracies, the public got a pretty accurate sense of near-Earth space physics. And we surely owe thanks to the TV show The Big Bang Theory for practically normalizing the concept of the physics nerd. The show has covered everything from the Mars rover to graphene to the most esoteric aspects of string theory. All of these efforts helped create a ready audience when CERN announced the Higgs particle measurement. And the media circus that formed was only too happy to move on to LIGO's observation of gravitational waves three years later.

Scientists, funding bodies, educators and the media alike need to carry this momentum forward. We live in a particular historic moment where this thirst for accurate science and fascination for astronomical events clashes with a strong movement rooted in scepticism towards scientists and research, from climate change to vaccines. When budgets get squeezed, it is all too easy to cut funding for outreach, but where are the scientists of tomorrow going to come from, and how can we tilt the public balance towards a more positive view of science? Spectacular shows that nature gives us for free, like the upcoming solar eclipse, are the perfect opportunity for this purpose, and we must welcome and support any initiative promoting them. 